\title{
A Survey on the Viewpoints of Nursing Managers on the Impact of Implementation of The Health System Development Plan on their Stress and Job Satisfaction in Hospitals
}

\author{
Research Article
}

\author{
Fatemeh Rezaei $^{1^{*}}$, Nasibeh Ramazannezhad ${ }^{2}$, Maedeh Faraji Douki ${ }^{3}$, \\ Mahsa Yaghoobzadeh Gatabi ${ }^{4}$, Mojgan Khodadadi ${ }^{3}$
}

\author{
1. Faculty Member, Department of Nursing, Islamic Azad University, Babol Branch, Babol, Iran. \\ 2. Anesthesiologist, Valiasr Hospital, Qaemshahr, Iran. \\ 3. Department of Nursing, Razi Hospital, Qaemshahr, Iran. \\ 4. Department of Nursing, Rouhani Hospital of Babol, Babol, Iran.
}

\begin{abstract}
Introduction: The aim of this study was to determine the viewpoint of nursing managers on the impact of implementation of the health system development plan on their stress and job satisfaction in hospitals of Babol University of Medical Sciences. Methods: This study is a descriptive study. In this study, the population of the study consisted of all metrons, supervisors and head nurses in hospitals affiliated to Babol University of Medical Sciences in 2017. Sampling method: Census; Sample size: In this research were 70 people; Research tool: Nursing job stress (Nursing Stress Scale) questionnaire (34 questions). The next questionnaire is the Spector's job satisfaction questionnaire (36 questions). In terms of validity, the questionnaires have been reviewed by experts and their reliability has been reported by Cronbach's alpha of 0.88 for this tool. Data analysis method: The data will be entered into SPSS version 22 using the statistical analysis. Findings: The results show that there is a significant relationship between the viewpoint of nursing managers on the impact of implementation of the health system development plan on their stress and job satisfaction in hospitals of Babol University of Medical Sciences in 2017 (Significant level less than 0.05). Conclusion: Despite the implementation of the health care development plan in nursing managers of hospitals affiliated to Babol University of Medical Sciences in 2017, their stress levels are more than average, and their job satisfaction is more than expected.
\end{abstract}

Keywords: Nursing Managers, Stress, Job Satisfaction.

\section{Introduction}

Job Satisfaction is often the cause of the conditions and relationships governing working environment and cultural factors (1). The Ministry of Health and Medical Education as the main custodian of the country's health system, in accordance with its duties and missions and upstream documents, in particular the 20-year vision document, health-related legislation in the fifth development plan, the health care development plan was launched in May 2014 with three approaches to protecting people's finances, providing access to health services, and improving service quality (2). The health care development plan includes 7 executive packages (reducing the amount of hospitalized patients in state hospitals, supporting the survival of physicians in deprived areas, the presence of specialist physicians residing in government hospitals, improving the quality of hoteling in government hospitals, the program for the promotion of normal delivery, the program of financial protection for

\section{*Corresponding Author:}

Fatemeh Rezaei

Department of Nursing,

Islamic Azad University,

Babol Branch,

Babol, Iran.

Email id: fatemehrezaei1360@yahoo.com patients with dementia, special and needy). These 7 packages are defined as 7 health service packages and are available on the official website of the Ministry of Health and Medical Education (3). The purpose of this plan was to increase the people's satisfaction in the health system in the first place, and although it is not directly addressed by the healthcare sector's satisfaction, but since the patient and his companion are often closely related to the personality and psychological and personnel components of the patient, the satisfaction of the employees has also been somewhat a goal in this evolution. One of the effective factors in employee productivity is job satisfaction (4). A high level of job satisfaction reflects a favorable organizational atmosphere, which can lead to an increase in employees' motivation and productivity (5). Job Satisfaction includes the needs, values, expectations and attitudes of individuals towards their jobs, and is affected by the work environment and the space they are working in. One of the occupations that are important for job satisfaction and mental health in general, is nursing. Regarding the stressfulness of the nursing profession, in recent years, there is an increase in the amount of stress in the workplace of this group of people, and this affects the health and safety of nurses and patients (6). According to the National Institute of Occupational Health and Safety, the person faces a stressful job when there is no coordination between job needs and his / her 
abilities and desires (7). In support of this issue, JacobSig in 2009 argues that accountability for occupational outcomes will increase the stress of decision making (8). Nursing managers experience a double burden of stress as being responsible for the management of all nurses' affairs and the clinical environment of the hospital.

Today, the issue of health is one of the main priorities of people's lives. In each health system, patients naturally expect to receive the required quality services at the right time (9). Hospitals and other health care centers are treated as an industrial unit, consisting of manufacturing factors such as capital, manpower, technology and management, which take steps to produce a product called the preservation, return and promotion of human health, and nurses including human resources of these centers (10). Nursing managers are also exposed to stress and burnout due to their role and there is more potential for them to leave, and so it is very important to ensure that they remain in their posts and that there is a shortage of nursing directors (11). Nurses' job satisfaction has become a vital challenge for the health system, so understanding job satisfaction is one of the effective factors in their job vacancies and it reduces the quality of work and irreparable harm to patients and themselves (12). The effect of job dissatisfaction on employee health, especially in recent years, has been the general concern of various societies. Understanding the effects of job dissatisfaction on the health of people from an economic perspective is also an important issue and plays an important role in the quality of professional and personal life of nurses and nursing directors (13). Also, according to Hall and et al., emotional responses are associated with a higher level of occupational stress. In other research, there is a direct and significant relationship between occupational stress and accountability (14). On the other hand, responding to its impact on competition, especially in the absence of resources, can increase job stress (15). Therefore, nursing managers who at any moment must be responsive to patients and their companions, as well as their other colleagues, such as nurses, doctors, and hospital managers, suffer a lot of stress. Job stress also affects the clinical competence of nurses and their managers, which increases the stress of nursing managers, because when other nurses do not have the appropriate clinical competence, the quality of service is reduced, which causes dissatisfaction with the patient and accompany him, and this is a stress for nursing directors. The results of various studies in this area have shown that one of the most fundamental missions of nursing managers is the continuous assessment of the clinical competence of nurses (16). Other factors affecting the job satisfaction of nurses and nursing managers are the physical environment of the workplace, because the physical space that is tailored to the patient's size and service is provided, the work process is better done, so nursing managers are less tense. In fact, by organizing in the physical structure of the work environment, it can reduce the time of doing things, increase the accuracy, improve the environment's safety, and control the work environment, increase productivity and decrease fatigue of nurses, which can reduce the stress of nursing managers (17). Therefore, management style, organizational culture, organization structure, nature of work, working conditions and type of communication with colleagues contributes to the formation of job satisfaction. The attraction and maintenance of employees' job satisfaction depends on factors such as a climate of trust, trust and decent work, analysis and appreciation of them, all of which lies in the organizational culture (18). Now, the question arises whether the implementation of this plan has had an impact on job satisfaction and reducing the stress of nursing managers or not? This is the same question that we asked in the present study to answer and provide information that serves as a guide and facilitator for the development of the health system of the country.

\section{Methods \\ Type of research}

This study is a descriptive study. In this study, the population of the study consisted of all metrons, supervisors and head nurses in hospitals affiliated to Babol University of Medical Sciences in 2017. The research sample in this study is equal to the research community.

\section{Criteria for entering the study}

All nursing managers who have been involved in the development of the health system in hospitals affiliated to Babol University of Medical Sciences. Exit criteria: Incomplete completed questionnaires will be deleted in less than $80 \%$ of the time.

\section{Sampling}

It was done by census. The sample size in this research is equal to the target community and will be around 70 people.

To investigate job stress, nursing job stress (Nursing Stress Scale) questionnaire will be used. This questionnaire contains 34 questions designed by Pritaft and Anderson in 1981, which provides job stress in nurses. Measurement scale is Likert 4 options. The lowest score from this questionnaire is 34 , and the highest score is 136 . The score between 34-68 show low stress, between 69-103 show medium stress and between 104-136 show high stresses. Validity and reliability of this questionnaire has been confirmed in various studies. For example, in the study of Hosseini et al. (2015), Cronbach's alpha coefficient was 0.93 and its external reliability was confirmed (19). The next questionnaire is the Spector's job satisfaction questionnaire. The questionnaire has 36 questions and focuses on 9 key factors. Each factor is measured in four terms. The options of this questionnaire are graded on the Likert scale of 5 degrees from the totally agreeable (score one) to completely opposite (score 5). Each subject can take a numerical job satisfaction between 36 and 180; this questionnaire was previously reviewed by Javadian et al. In 2014 in terms of validity and reliability, and they have reported 0.86 for Cronbach's alpha for this tool (20). 
Data analysis method

The data will be entered into SPSS version 22 using statistical software. Then, using descriptive variables, we extracted the variables and used the inferential statistics of the comparisons. Dispersion indicators such as average, frequency distribution, standard deviation are used. For the mean and other items mentioned for statistical analysis, one-sample ttest was used the 95\% confidence interval $(\mathrm{P} \leq 0.05)$ would be a significant level in this study. Also descriptive statistics were used in this table and there is no need for the mean and other mentioned items.

\section{Findings}

Table 1. Frequency distribution of demographic information percentage of nursing managers in hospitals of Babol University of Medical Sciences

\begin{tabular}{|c|c|c|}
\hline \multirow{2}{*}{ Gender } & Male & 0.67 \\
\cline { 2 - 3 } Marital status & Female & 0.33 \\
\hline \multirow{3}{*}{ Degree of education } & Single & 0.16 \\
\cline { 2 - 3 } & Married & 0.84 \\
\cline { 2 - 3 } & Bachelor & 0.91 \\
\cline { 2 - 3 } Work experience & Masters & 0.9 \\
\hline \multirow{4}{*}{ P.H.D } & 0 \\
\cline { 2 - 3 } & $\begin{array}{c}\text { Under 10 years } \\
\text { old }\end{array}$ & 0 \\
\cline { 2 - 3 } Academic Rank & $\begin{array}{c}\text { Over 20 years } \\
\text { old }\end{array}$ & 0.64 \\
\hline \multirow{3}{*}{ Contractual } & 0.36 \\
\cline { 2 - 3 } & Conventional & 0.16 \\
\cline { 2 - 3 } & Official & 0 \\
\cline { 2 - 3 } & \multicolumn{2}{|c|}{0.84} \\
\hline
\end{tabular}

The above table shows that according to the sample, nursing managers are more than 0.67 from male groups. It can also be concluded that in the present study $84 \%$ of them are married, and it can be said that most nursing managers have a bachelor's degree, and those with a work experience of 10 to 20 years have the highest age, it can also be concluded that in this research, most managers with $84 \%$ have formal employment status.

Considering the research questions in the field of occupational stress in managers of hospitals of Babol University of Medical Sciences with an average (3.03), we conclude that the stress base has been seen in high and average nurses (due to the fact that the average stress in hospital managers is above the 3 ) and most of their stressors come back to the environment and colleagues' behavior and this stress is seen in all environmental, organizational and individual factors, so that managers in the questions of the questionnaire respond to all the questions of stress in a medium and high level and indicate that there is a moderate to high level stress among them.
Table 2. T-test stress assessment of hospital managers in Babol University of medical sciences in health plan

\begin{tabular}{|c|c|}
\hline Variable & Stress in Hospital Managers \\
\hline Probability value & 0 \\
\hline Test statistic & 39.33 \\
\hline Degrees of freedom & 58 \\
\hline mean & 3.03 \\
\hline SD & 1.06 \\
\hline Min & 1 \\
\hline Max & 5 \\
\hline
\end{tabular}

According to the above table data, since the probability value is 0.000 and less than 0.05 , so at a significant level 0.05 the zero hypotheses are rejected. Since the average of 3.03 is more than 3 , it can be concluded that the occupational stress of nursing managers achieved a higher score than expected. Consequently, despite the implementation of the health care development plan in nursing managers of hospitals affiliated to Babol University of Medical Sciences in 2016, their stress levels are still more than average. According to the research questions in the field of job satisfaction of hospital managers in Babol University of Medical Sciences with an average (3.71), we conclude that the field of job satisfaction in nurses has been seen in a high number of options. (Considering that the average level is assumed to be three in the Likert spectrum, therefore, we assume three basic data bases based on the average job satisfaction in hospital managers above 3 , which is why there is a good level) which indicates satisfactory satisfaction in managers.

Table 3. T-test of job satisfaction of hospital managers of Babol University of medical sciences in health plan

\begin{tabular}{|c|c|}
\hline Variable & $\begin{array}{c}\text { Job Satisfaction in } \\
\text { Hospital Managers }\end{array}$ \\
\hline Probability value & 0 \\
\hline Test statistic & 14.19 \\
\hline Degrees of freedom & 58 \\
\hline mean & 3.71 \\
\hline SD & 1.12 \\
\hline Min & 1 \\
\hline Max & 5 \\
\hline
\end{tabular}

According to the above table data, since the probability value is 0.000 and less than 0.05 , so at a significant level 0.05 the zero hypotheses are rejected. Since the average of 3.71 is more than 3 , it can be concluded that the job satisfaction of nursing managers has exceeded the expected level. As a result, after implementation of the health system development plan in nursing managers of hospitals affiliated to Babol University of Medical Sciences in 2016, their job satisfaction is more than expected. 


\section{Conclusion and Discussion}

In relation to goal 1, " Determining the level of stress after the implementation of the health system development plan in nursing managers of hospitals affiliated to Babol University of Medical Sciences in 2016" indicates that implementation of the healthcare development plan in nursing managers of Babol University of medical sciences hospitals affects job stress. The results of the study are in line with Assadi's (2017); Pak (2011); Flanner (2015); Kimit (2014); Sharon (2008); their research findings showed that nursing jobs are associated with a high degree of stress due to their nature and due to their relationship with the lives and lives of other occupations, it is important to have a nursing job. One of the important issues in this health plan is the lack of nurses at nursing stations. Also, according to the responses of the research samples, the inappropriate condition of paying nurses, shift work and shift work, as well as working pressure, cause a double burden on nurses and also reduce the emotional relationship between them and lead to an increase in occupational stress (21).

In relation to goal 2, "Determining the level of job satisfaction after the implementation of health system development plan in nursing managers of hospitals affiliated to Babol University of Medical Sciences in 2016" indicates that implementation of the healthcare development plan in nursing managers of Babol University of medical sciences hospitals affects job satisfaction. The results of the study are in line with Habibi's studies (2015); Laurber (2012); Mousavi (2015); Abbaschian (2012); Mirfarhadi (2014); their research findings showed that nurses' dissatisfaction can threaten physical, mental and quality of life, impede the achievement of their goals, their individual and social development, and reduce individual interest in attending work. In Abbaschian et al. Study, welfare facilities, satisfaction with the work environment and improvement of salaries and benefits were the most important factors affecting the satisfaction of nurses (22). The results of Mousavi's study in Tehran indicated that nurses' satisfaction was in a good level and had the highest satisfaction with the sense of responsibility in work and the lowest satisfaction with salaries and wages (23). The study by Mastan et al., aimed at assessing the job satisfaction of nurses in Hormozgan teaching hospitals, showed that the satisfaction of nurses was moderate (18.3\%) (24).

Overall, job stress in nursing managers scored more than expected levels, consequently, despite the implementation of the health system development plan in nursing managers of Babol University of medical sciences hospitals in 2016, their stress levels are more than average, Also, job satisfaction of nursing managers has a higher score than expected, As a result, after implementation of the health system development plan in nursing managers of hospitals affiliated to Babol University of Medical Sciences in 2016, their job satisfaction is more than expected.

\section{References}

1. Pashib M, Seyedmoharrami I, Mohammadi S, Maryam T. Stress management group therapy is job satisfaction and general health in nurses. Journal of Torbat Heydariyeh University of Medical Sciences. 2015; 3 (2): 7-1.

2. Pirooz B, Mohamadi Bolban Abad A, Moradi G. Assessing the Health System's Responsiveness after the Implementation of the Health System Reform: A Case Study of Sanandaj, 2014-2015. Iranian Journal of Epidemiology. 2016; 11 (4): 1-9.

3. A survey on patient and patient's companion satisfaction for health reform plan in the Shahid Mostafa Khomeini Hospital of Ilam in 2015. Nursing of the vulnerable journal. 2016; 2 (5): 27 39.

4. Jafary E, Kamarzarin H, Kordmirza E, Sefizadeh V. The role of spiritual well-being and coping skills in the prediction of job satisfaction in nurses. Journal of Clinical Nursing and Midwifery. 2015; 4 (1): 508.

5. The impact of the implantation of the Health Care Reform Plan in patients paid out of the pocket in selected Public hospitals in Isfahan. Journal of medical council of Islamic republic of Iran. 2015; 33 (3): 0-.

6. Hosein MA, Asadi Nejad H, Dalvandi A. The Effect of Implementing Workplace Organization Technique is Nurses' Job Satisfaction. MedicalSurgical Nursing Journal. 2016; 5 (1): 57-63.

7. Hosseini Z, Aghamolla $\mathrm{T}$, Moein B, Hazavehei MM, Moghimbeigi A. The effect of health education program on female nursing stress. J Health Syst Res. 2015; 11 (1): 43-52.

8. Khosrozadeh M, Hosseini M, Kashaninia Z, Sedghi Goyaghaj N, Amini M. The correlation between organizational justice and job satisfaction among nurses. Journal of Health Promotion Management. 2016; 5 (2): 10-9.

9. Dehghan A, Mirjalili MR, Zare Mehrjardi MH, Maliheh R, Samiyezargar A, Kazemein SK. Performance of the Health Care System Reform Plan From the Perspective of University Hospitals Executives in Yazd Province in 2015. Management Strategies in Health System. 2016; 1 (1): 43-9.

10. Sirati-Nir M., Khaghanizadeh M. The Study of Relationship Stress and Efficiency among Nursing Managers in a Selected Hospital. Journal of Military Medicine. 2003; 5 (1): 33-7.

11. Sahebzadeh M, Karimi S, Hosseini M, Akhtar Danesh G, Hosseini S. Job Burnout of Nursing Administrators and Chief Executive Officers in the University Hospitals and its Relation to Their Demographic Features. Health Information Management. 2011; 7 ((Special Issue)): 648.

12. Gharibi F, Janati A, Farajollah Beiknor, M, Amini Daghalian B. A Survey of Health System Reform Taleqani Hospital from the Experiences of Managers and Nurses of Tabriz. Depiction of Health. 2015; 6 (1): 1-10.

13. Ghasemzadeh-Alishahi A, Rabiei M, KazemzadehBeytali M. Relationship of Individual 
Accountability with Job Stress and Work Deviant Behavior among Nurses. Journal of Clinical Nursing and Midwifery. 2015; 4 (1): 27-38.

14. Komeil-Sani M, Etemad A, Boustan H, Bahrain M, Hakim A. The relationship between nurses' clinical competency and job stress in Ahvaz University Hospital, 2013. Journal of Clinical Nursing and Midwifery. 2015; 4 (1): 39-49.

15. Mohammadzadeh A, Ghorbani K, Jafari E. Assessing the Role of Personality Traits and Job Engagement in Predicting Nursing Stress among Orthopedic Nurses in Shohada Hospital of Tabriz. Hospital 2016; 15 (1): 69-76.

16. Habibi h, Mooghal A, Habibi F, AHMADI m. Comparative study of the relationship between job satisfaction and burnout among Nurses in Public and Private Hospitals in Shiraz City in 2012. Hospital. 2015; 14 (3): 119-26.

17. Mehdad A, Asadi A, Golparvar M. The Moderating Role of Religious Beliefs is the Relationship between Nurses' Job Stress and General Health. Knowledge \& Research in Applied Psychology. 2015; 17 (2): 15-25.

18. Narimani M, Salehi M, Kazemi N. The Effectiveness of Dialectical Behavior Therapy is the Stress Reduction and Increasing Self-Efficacy among Nurses. Journal of Health and Care. 2015; 17 (4): 270-80.
19. Lee P, Miller MT, Kippenbrock TA, Rosen C, Emory J. College nursing faculty, job satisfaction and retention: A national perspective. Journal of Professional Nursing. 2017

20. Murrells T, Clinton M, Robinson S. Job satisfaction in nursing: validation of a new instrument for the UK. Journal of Nursing Management. 2005; 13 (4): 296-311.

21. Abaschian R, Avazeh A, Rabie Siahkali S. Investigation of nursing job satisfaction and related factors in hospitals affiliated to Zanjan University of Medical Sciences. JNMS 2012; 1 (1): 17-24.

22. Lorber M, Skela Savič B. Job satisfaction of nurses and identifying factors of job satisfaction in Slovenian hospitals. Croatian medical journal. 2012; 53 (3): 263-70.

23. Mastaneh Z, Mousel L, Zamani M, Boromand E, Dadipoor $\mathrm{S}$, Beizaei $\mathrm{F}$, et al. Investigation of nursing job satisfaction in university hospitals affiliated to the Hormozgan University of Medical Sciences. Bimonthly Journal of Hormozgan University of Medical Sciences. 2014; 18 (3): 2606.

24. Mousavi SS, Movahed Rad S. The study of job satisfaction among nurses in Tehran's military hospitals in 2014. Journal of Nursing and Physician in War. 